\title{
Erratum: Detecting dark matter through dark photons from the Sun: Charged particle signatures [Phys. Rev. D 93, 115036 (2016)]
}

\author{
Jonathan L. Feng, Jordan Smolinsky, and Philip Tanedo
}

(Received 9 October 2017; published 6 November 2017)

DOI: $10.1103 /$ PhysRevD.96.099903

The code used to produce the final results and plots in this paper contained a typographical error in the dark photon decay length. None of the equations in the paper needs to be modified, but Figs. 5 and 7-10 should be replaced by the updated versions included here.

In accordance with the modified results of other papers in the sequence, the region of parameter space accessible to a search for dark photons coming from the center of the Earth described in the Introduction should be adjusted to read, "As an example, in currently unconstrained regions of parameter space with dark matter masses $100 \mathrm{GeV} \lesssim m_{X} \lesssim 10 \mathrm{TeV}$, dark photon masses $m_{A^{\prime}} \sim \mathrm{MeV}-\mathrm{GeV}$, and kinetic mixing parameters $10^{-10} \lesssim \epsilon \lesssim 10^{-8}$, this scenario predicts up to thousands of TeV-energy $e^{+} / e^{-}, \mu^{+} / \mu^{-}$, and hadron pairs from the center of the Earth streaming through the IceCube detector each year." Similarly, the region of parameter space explored in this paper and discussed in the Introduction should read, "Including these effects, we show that AMS can discover dark matter through the dark sunshine signal for parameters $100 \mathrm{GeV} \lesssim m_{X} \lesssim 10 \mathrm{TeV}, m_{A^{\prime}} \sim \mathcal{O}(100) \mathrm{MeV}$, and $10^{-10} \lesssim \epsilon \lesssim 10^{-8}$."
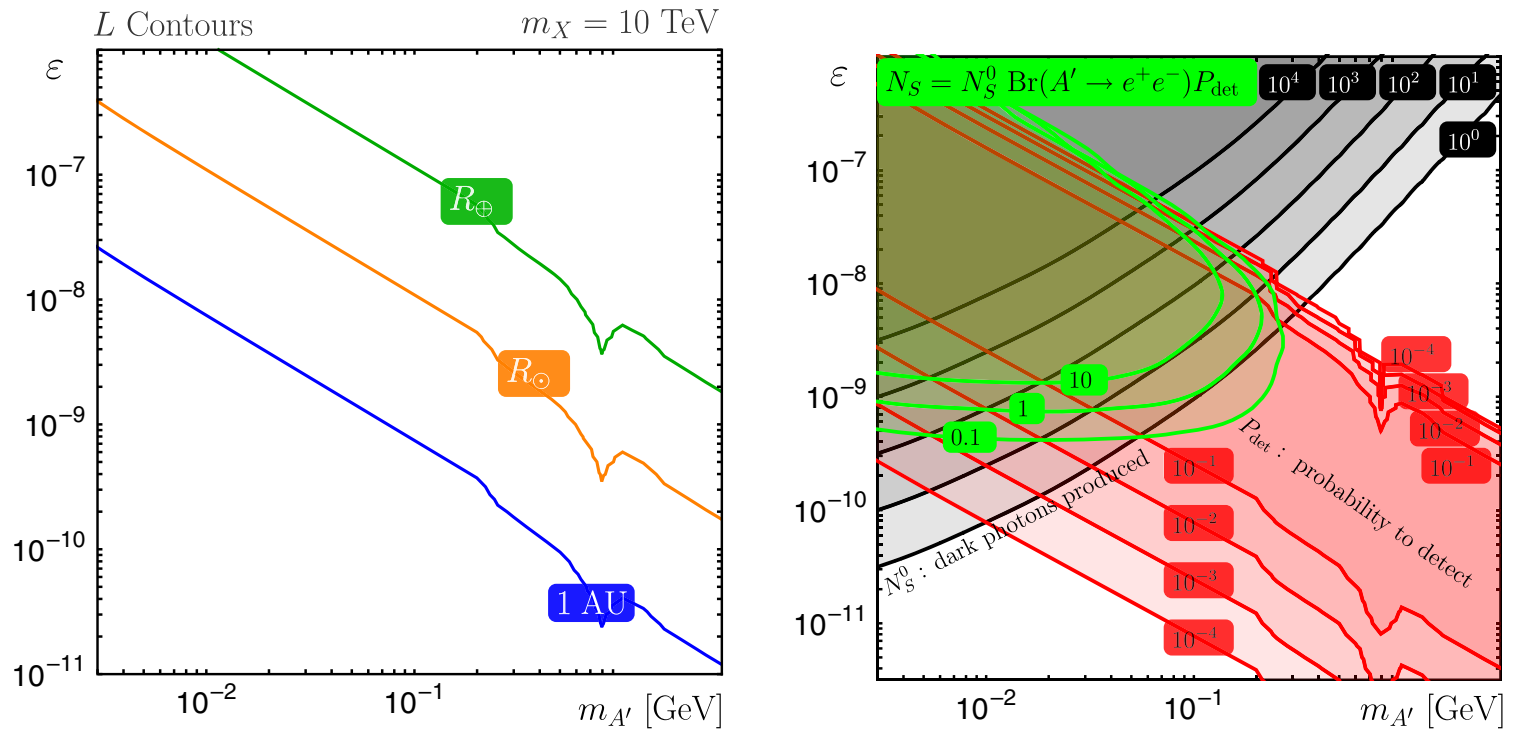

FIG. 5. Left: Contours of fixed decay length $L=R_{\oplus} \simeq 6400 \mathrm{~km}, R_{\odot} \simeq 7.0 \times 10^{6} \mathrm{~m}$, and 1 au in the $\left(m_{A^{\prime}}, \epsilon\right)$ plane for $m_{X}=10$ TeV. The dip at $775 \mathrm{MeV}$ comes from resonant $A^{\prime}$ decays via $\rho$ mesons mixing. The decay lengths shape the probability contours through Eq. (53). Right: Factors determining the signal reach for dark sunshine searches at AMS for live time $T=3$ years and $m_{X}=10$ TeV. BLACK: $N_{S}^{0}$, the number of dark photons produced when the Sun is in AMS's field of view. RED: $P_{\text {det }}^{\max }$, the optimal positron detection probability at each point in the $\left(m_{A^{\prime}}, \epsilon\right)$ plane. GREEN: $N_{S}$, signal region reach. In most of this plane, $\operatorname{Br}\left(A^{\prime} \rightarrow e^{+} e^{-}\right)=1$, and so the green contours are products of the red and black contours. For example, the $N_{S}=1$ contour passes through the intersection of the $N_{S}^{0}=10$ and $P_{\operatorname{det}}^{\max }=0.1$ contours. 

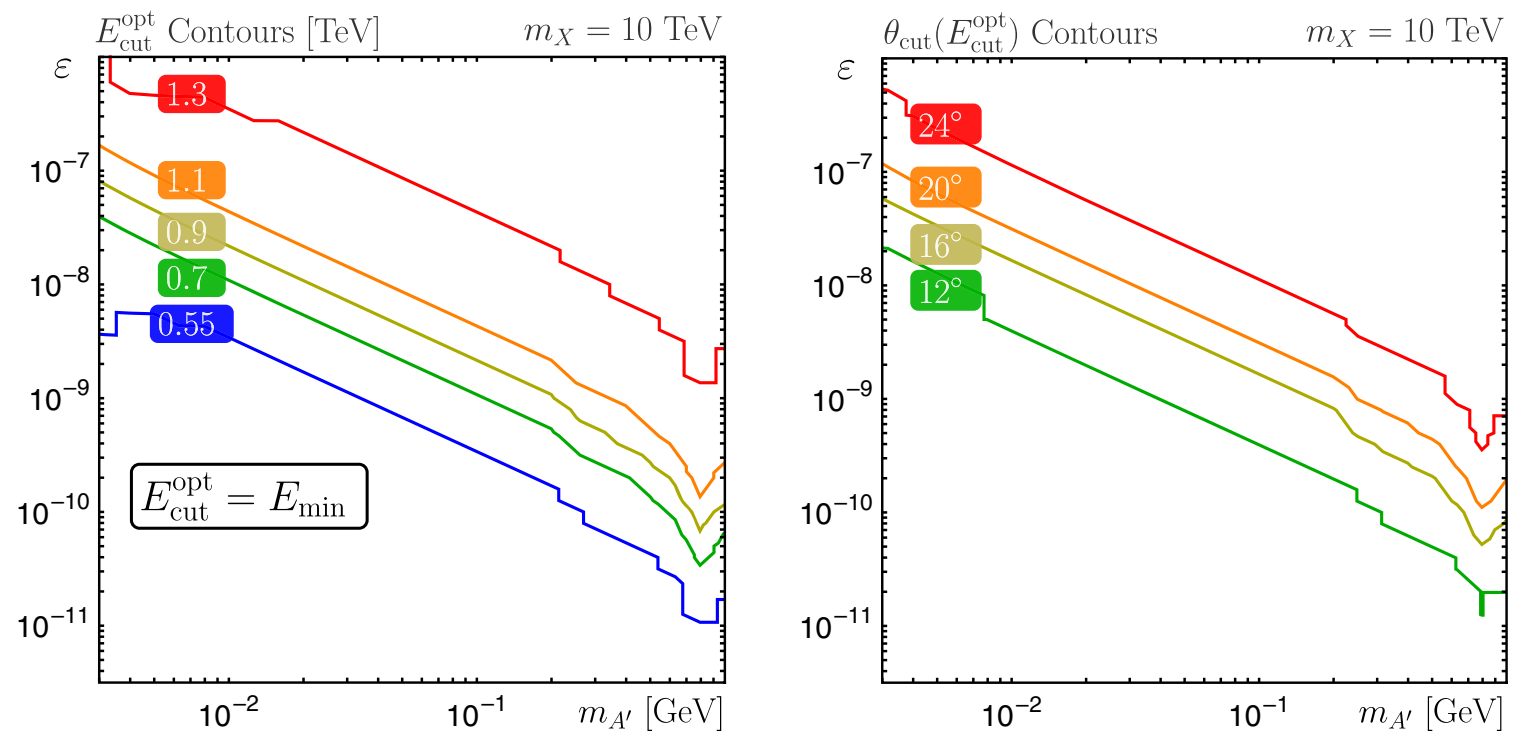

FIG. 7. Left: Contours of $E_{\mathrm{cut}}^{\mathrm{opt}}$, the value of $E_{\mathrm{cut}}$ that maximizes the probability $P_{\mathrm{det}}$ in the $\left(m_{A^{\prime}}, \epsilon\right)$ plane for $m_{X}=10 \mathrm{TeV}$. In the region below lowest plotted contour, $550 \mathrm{GeV}, E_{\text {cut }}^{\text {opt }}>E_{*}$ so that the $P_{\text {det }}^{B}$ term in Eq. (49) vanishes-the solar magnetic field does not affect the choice of cuts-and $E_{\text {cut }}^{\mathrm{opt}}=E_{\min }$. Right: Contours of the corresponding values of $\theta_{\text {cut }}$ from Eq. (39). 

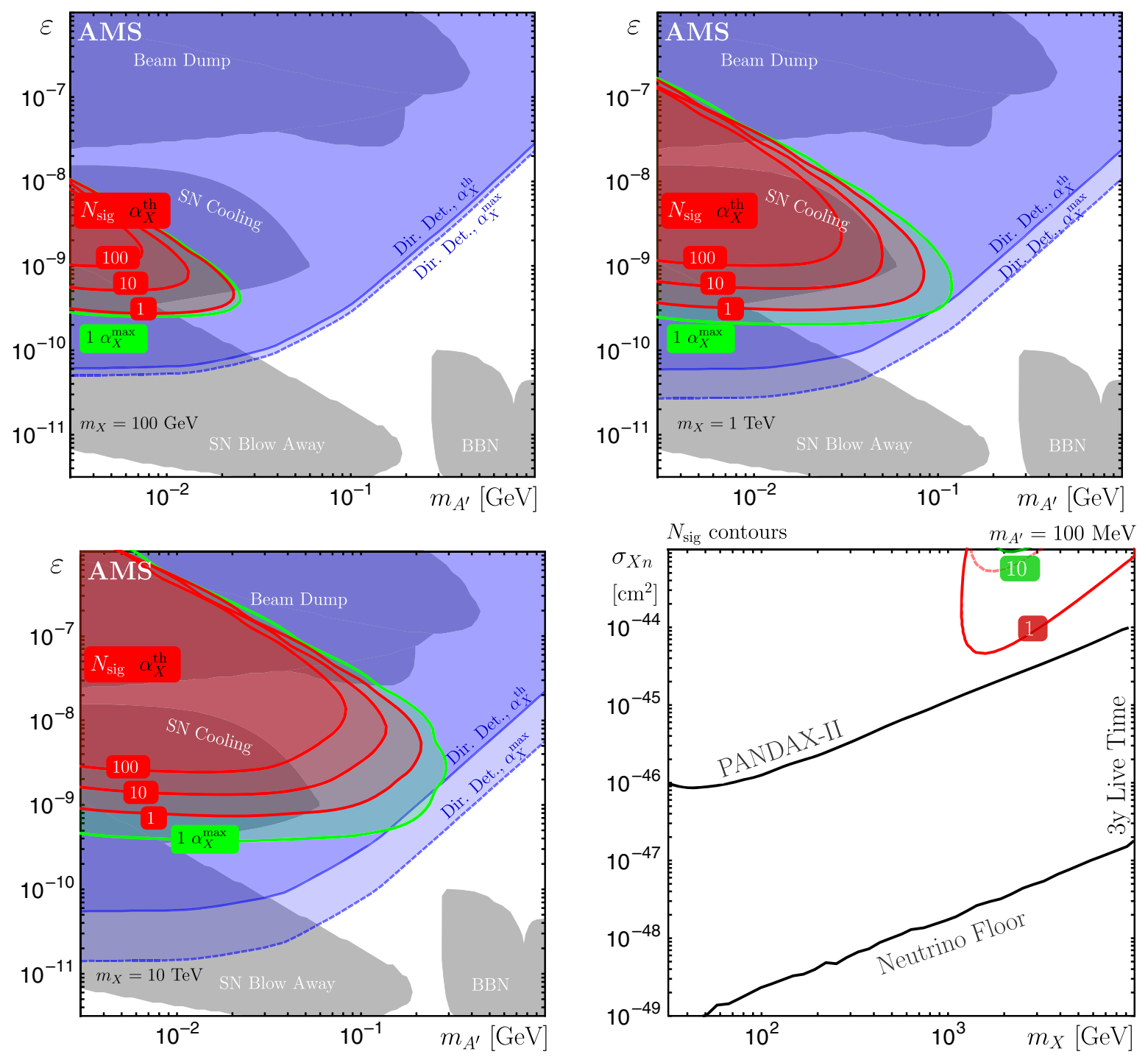

FIG. 8. Top and bottom left: RED: Region probed by AMS search with indicated number of signal events $N_{S}$ for $m X=100 G e V$, $1 \mathrm{TeV}, 10 \mathrm{TeV}$, assuming $N_{B}=1$ background event and $T=3$ years live time in the $\left(m_{A^{\prime}}, \varepsilon\right)$ plane. The dark sector fine-structure constant $\alpha_{X}$ is set by requiring $\Omega_{X} \simeq 0.23$. GREEN: The $N_{S}=1$ reach for $\alpha_{X}=\alpha_{X}^{\max }$, the maximal allowed coupling from CMB bounds [1-3], as written in Eq. (6). BLUE: Current bounds from direct detection [4,5]. GRAY: Regions probed by other dark photon searches discussed in Sec. III. Bottom right: Comparison of indirect and direct detection sensitivities in the $\left(m_{X}, \sigma\right)$ plane for $m_{A^{\prime}}=100 \mathrm{MeV}$. RED: $N_{S}=1$ signal event contours for $\alpha_{X}=\alpha_{X}^{\text {th }}$ (solid) and $\alpha_{X}^{\max }$ (dashed). GREEN: Same, but for $N_{S}=10$. The direct detection bounds are from the PANDAX-II experiment [5]; note that in this regime the pointlike interaction limit is valid; this is not the case for the low $m_{A^{\prime}}$ region $[4,6,7]$. Also shown is the "neutrino floor," where coherent neutrino scattering affects direct detection experiments [8]. 

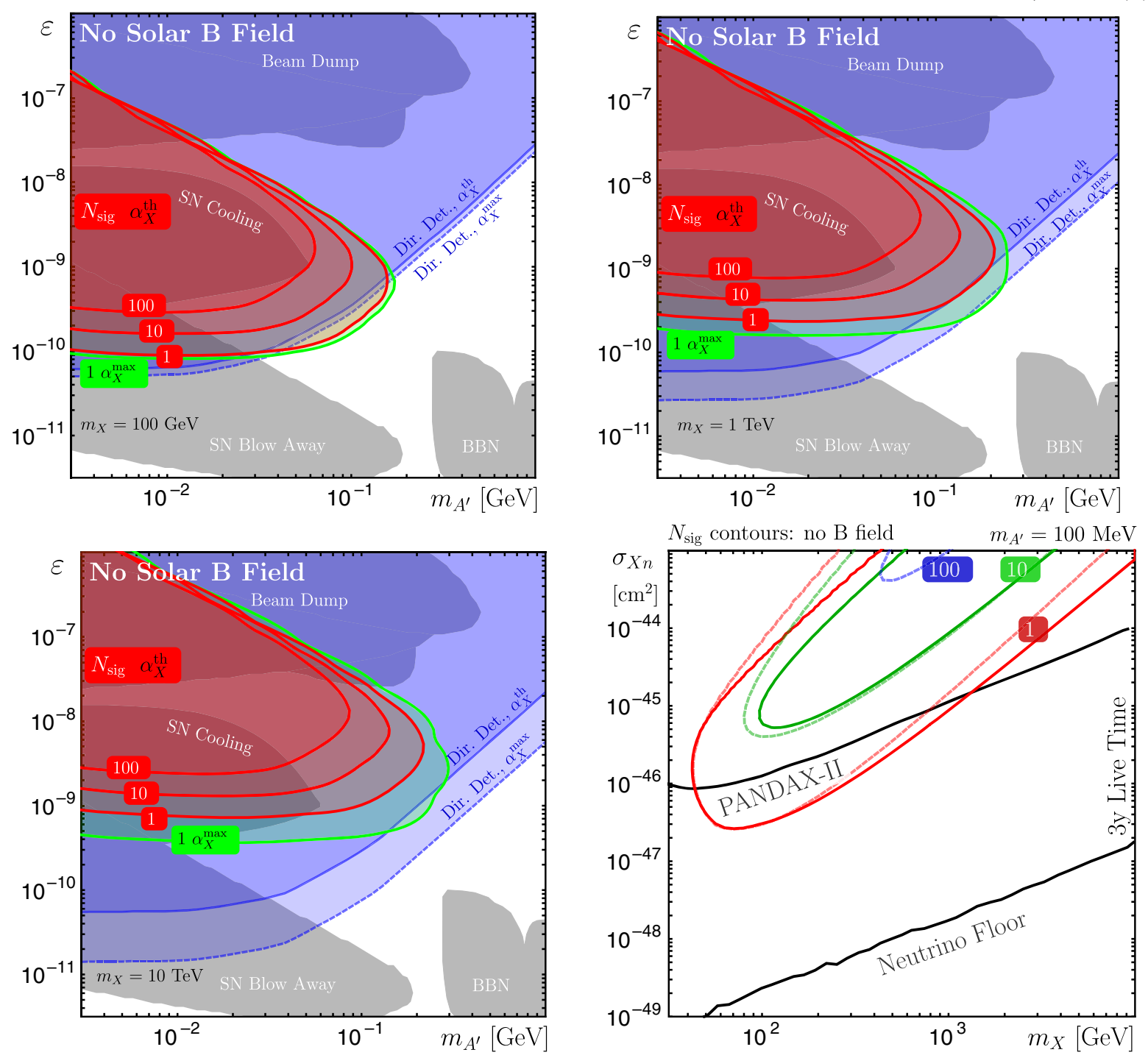

FIG. 9. Same as Fig. 8 but with no solar $B$ field. Comparing to Fig. 8, one sees that a large fraction of potential signal positrons is deflected for lighter dark matter masses. 

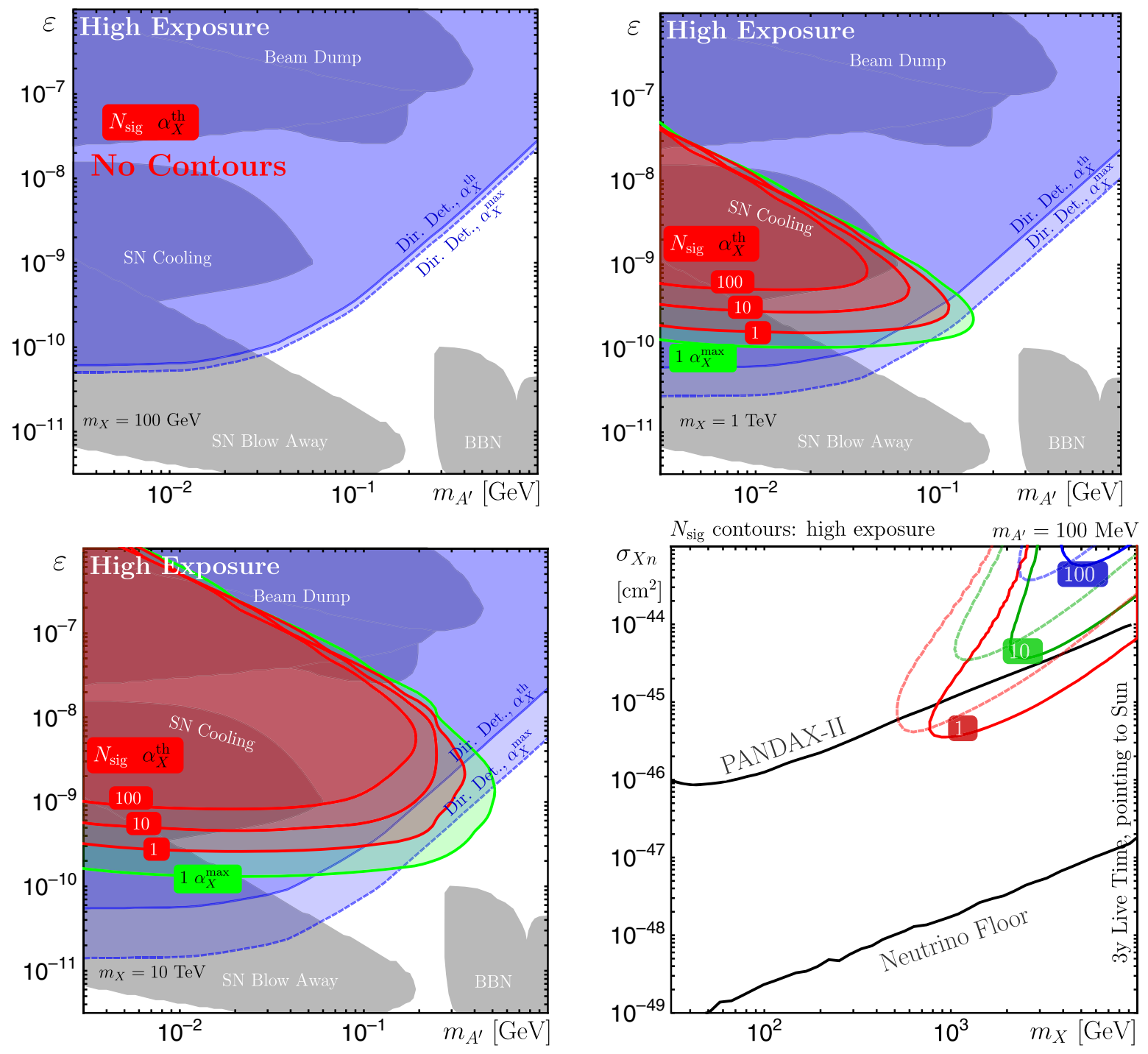

FIG. 10. Same as Fig. 8 but for a hypothetical high-exposure experiment with $\xi_{\odot}^{\text {high }}=80 \xi_{\odot}$. The condition of a single background event $N_{B}=1$ in Eq. (39) sets such strong cuts that there are no signal positrons for $m_{X} \lesssim 500 \mathrm{GeV}$.

We are grateful to Adam Green for bringing the error to our attention.

[1] J. A. Adams, S. Sarkar, and D. W. Sciama, CMB anisotropy in the decaying neutrino cosmology, Mon. Not. R. Astron. Soc. 301, 210 (1998).

[2] X. -L. Chen and M. Kamionkowski, Particle decays during the cosmic dark ages, Phys. Rev. D 70, 043502 (2004).

[3] N. Padmanabhan and D. P. Finkbeiner, Detecting dark matter annihilation with CMB polarization: Signatures and experimental prospects, Phys. Rev. D 72, 023508 (2005).

[4] E. Del Nobile, M. Kaplinghat, and H. -B. Yu, Direct detection signatures of self-interacting dark matter with a light mediator, J. Cosmol. Astropart. Phys. 10 (2015) 055.

[5] X. Cui et al. (PandaX-II Collaboration), Dark Matter Results From 54-Ton-Day Exposure of PandaX-II Experiment, arXiv:1708.06917.

[6] M. Kaplinghat, S. Tulin, and H. -B. Yu, Direct detection portals for self-interacting dark matter, Phys. Rev. D 89, 035009 (2014).

[7] H. An, M. Pospelov, J. Pradler, and A. Ritz, Direct detection constraints on dark photon dark matter, Phys. Lett. B 747, 331 (2015).

[8] J. Billard, L. Strigari, and E. Figueroa-Feliciano, Implication of neutrino backgrounds on the reach of next generation dark matter direct detection experiments, Phys. Rev. D 89, 023524 (2014). 